\title{
The emerging specialty of cardiothoracic surgical critical care: The leadership role of cardiothoracic surgeons on the multidisciplinary team
}

Nevin M. Katz, MD

From the George Washington University, the Washington Institute of Thoracic and Cardiovascular Surgery, and the Foundation for the Advancement of Cardiothoracic Surgical Care, Washington, DC.

Nevin Katz, MD, reports that he is paid to direct conferences on cardiothoracic surgical care.

Received for publication April 30, 2007; revisions received June 13, 2007; accepted for publication July 5, 2007.

Address for reprints: Nevin M. Katz, MD, 2175 K Street, NW, Suite 300, Washington, DC 20037 (E-mail: nevinkatz@aol.com).

J Thorac Cardiovasc Surg 2007;134:1109-11 $0022-5223 / \$ 32.00$

Copyright (C) 2007 by The American Association for Thoracic Surgery

doi:10.1016/j.jtcvs.2007.07.032
$\mathrm{I}$ $\mathrm{t}$ is apparent that the increasing complexity of cardiothoracic surgical (CTS) cases requires a new level of critical care performance. Although the cardiothoracic (CT) surgeon has traditionally provided this care with the assistance of residents and fellows, the organization of critical care is changing. A new system of multidisciplinary CTS critical care is emerging, for reasons described below. I believe that the CT surgeon is uniquely positioned to have a leadership role on the multidisciplinary team and to coordinate this new system of care.

With the changes in house staff working hours and the corresponding decreasing role of surgical residents and fellows in the care of CTS patients, physician assistants and nurse practitioners are having a greater role in minute-to-minute clinical decision making and protocol development. It is important that this valuable participation of paramedical personnel be properly integrated into CTS critical care programs. The surgeon is uniquely qualified to coordinate and to assist in the training and certification of paramedical personnel as they assume this expanded role in the critical care of CTS patients.

The situation, however, is more complicated in several regards. The relative roles of CT surgeons and intensivists in the CTS critical care unit have become unclear. Safety remains an issue in health care and includes the field of critical care. New protocols have been instituted to address safety issues in the intensive care unit. ${ }^{1}$ A variety of studies in the critical care literature have led to new concepts of management with improvements in mortality and morbidity. Examples include the treatment of sepsis, ${ }^{2}$ the management of patients with ventilator-associated pneumonia, ${ }^{3}$ and the use of lung-protective ventilation strategies in acute lung injury. ${ }^{4}$ Some concepts such as the importance of tight glucose control in the critical care patient have appeared in the CT literature as well. ${ }^{5}$ It is clearly important that the new developments in the general field of critical care be applied to patients receiving intensive care after CT surgery. Overall, as has been widely recognized, it is important that the latest scientific studies be applied correctly, using what has been described as "evidence-based medicine."

\section{CTS Critical Care: A Specialty?}

In a sense, CTS critical care is inherent to the specialty of CT surgery. The CT surgeon provides critical care to his or her patients because of the nature of the surgery. This is the case for a substantial proportion of patients, as reflected in their receiving care early postoperatively in intensive care units. From the beginning of a surgeon's training in this specialty, experience providing critical care is an integral part of the residency or fellowship program.

However, with the increasing complexity of such care and the involvement of critical care physicians and allied health care professionals, the question arises whether the critical care of CTS patients is appropriately considered part of general critical care or whether it is, indeed, a specialty itself, distinct from general critical care. In support of the latter, it is noted that the clinical situations of CT surgery, including cardiopulmonary bypass, cardiac reconstructive procedures, pulmonary resections, and esophageal surgery, create unique physiologic changes that require special considerations. In addition, complications associated with CT procedures 
are indeed unique in medical practice. Accordingly, in my opinion, CTS critical care qualifies as a specialty within the frameworks of CT surgery and critical care.

\section{CTS Critical Care: A Multidisciplinary Specialty}

It is clear from clinical practice that CTS critical care requires a detailed knowledge of multiple medical and surgical disciplines. Correspondingly, a team of health care professionals provides CTS critical care. They include CT surgeons, critical care physicians and anesthesiologists, interventionists, other subspecialty physicians, critical care nurses, physician assistants, nurse practitioners, perfusionists, respiratory therapists, nutritionists, and pharmacists.

Therefore, in defining the breadth of the specialty, to provide for its growth and the education of the entire team, it is important to recognize that the specialty is, indeed, multidisciplinary.

\section{Challenges of CTS Critical Care in This Era}

In this era, the increased acuity of patients undergoing CT surgery and the range and complexity of the procedures involved place increasing and changing demands on the expertise of the CTS critical care team. For example, average patient age has progressively increased, and patients, accordingly, continue to have more associated medical conditions. In many cases, the disease for which the patient undergoes operation is more advanced than those in earlier experiences in the specialty of CT surgery.

Another factor that challenges the expertise of the CTS health care team is the ongoing evolution in maximally supportive technology used both during and after the operation. This technology includes advanced ventilation systems, extracorporeal life support, ${ }^{6}$ ventricular assist devices, and continuous renal replacement therapies. ${ }^{7}$ The complexity of the equipment and the on-going improvements in design demand detailed protocols and knowledgeable, experienced bedside professionals. In a sense, the technological environment of the CT operating room has been extended to the CTS intensive care unit.

An additional challenge to the CTS critical care team is the integration of new pharmaceutical agents and combinations of pharmaceuticals into management protocols. Examples of evolving approaches to important clinical issues in CTS critical care include the use of inhaled prostacyclin and nitric oxide for severe pulmonary hypertension ${ }^{8}$ and the use of arginine vasopressin for postcardiotomy vasodilatory shock. ${ }^{9}$ New pharmaceuticals have the potential for greater specificity and greater potency. On the other hand, new products, with their own idiosyncrasies, challenge the team to provide a safe introduction of the associated management protocols.

New bedside technologies available for CTS critical care further challenge the CTS team. Some of these new devices and techniques importantly enhance diagnosis and increase the safety of care. Examples are the use of advanced hemodynamic catheter devices, impedance cardiography, and bedside portable ultrasonography. The latter two technologies provide valuable information in the critical care unit while having the advantages for the patient of being noninvasive. Overall, new technologies provide the means to extend the inherent precision of CT surgery from the operating room to the critical care unit.

\section{Conferences Focused on the Specialty of CTS Critical Care}

Given the complexity of the field of CTS critical care and the multidisciplinary nature of the critical care team, it is important that the members of the team share a broad understanding of the field, including its latest advances. This approach of ensuring that members of the team are "on the same page" provides the optimal environment for coordination and implementation of management procedures and protocols. In this regard, successful annual "Cardiothoracic Surgical Critical Care" conferences focused on the specialty were instituted in 2004.

CT surgeons have played an important role in creating and planning the conferences and participating as faculty. The central goal of the conferences has been to provide the means for a dialogue among members of CTS critical care teams, including experts and world authorities, to further advance knowledge and expertise in this complex field. Topics have been chosen to highlight new concepts, innovative technologies, and current issues and controversies in CTS critical care. The programs have included symposiums dedicated to the important issues of "patient safety" and "the critical care environment" for the patient and the health care professional. As part of the development of these conferences, a multidisciplinary board of advisors, now composed of 33 multidisciplinary specialists, 9 of whom are CT surgeons, has been created to guide and participate in the program agendas of the conferences.

\section{The Future of CTS Critical Care and the Role of the CT Surgeon}

It is clear that the CT surgeon should have an important leadership role on the multidisciplinary team. It is key that one of the leaders of the team caring for a CTS patient understands the details of the individual's operation and the anticipated response to the procedure(s). The surgeon has the best perspective of this. It is fundamental that the patient has placed his life in the hands of the CT surgeon and, accordingly, expects the surgeon to have an important role in the postoperative care, working closely with critical care specialists. This approach preserves the essential element of continuity of care.

It is important for thoracic surgeons to stay in the forefront as leaders in the specialty of CTS critical care. Mul- 
tidisciplinary conferences provide an opportunity for CT surgeons to stay updated in this rapidly evolving field and support their leadership role on the multidisciplinary team. This perspective gives CT surgeons the background to have an important role in the coordination and training of critical care personnel.

A number of established CT surgeons are expressing an interest in making a transition to the specialty of CTS critical care as a supplementary or primary professional activity. Questions have been raised about the need for certification. It would seem that a CT surgeon who has been certified by the American Board of Thoracic Surgery is qualified to provide the critical care of CTS patients, as this is inherent in the discipline. Nevertheless, with evolving critical care knowledge and technology, there is the need for updates of knowledge and expertise and perhaps additional certifications.

In my opinion, the formal study of CTS critical care should be a part of thoracic surgery training programs. Such training should come from CT surgeons as well as from anesthesiologists and critical care specialists in the multidisciplinary setting. In regard to the recent interest on the part of a number of certified CT surgeons to obtain certification in critical care, I would suggest that the American Board of Thoracic Surgery consider offering certification in the specialty of CTS critical care, as well as in classic thoracic surgery.

In summary, a new specialty of CTS critical care has emerged as a reflection and a result of the unique physiologic changes and unique complications associated with $\mathrm{CT}$ surgery and the rapid evolution in the care of CTS patients. The specialty crosses the disciplines of medicine and surgery and involves a spectrum of health care professionals. CT surgeons have an important leadership role in the specialty as they maintain continuity of patient care and work closely with the multidisciplinary team.

\section{References}

1. Curtis JR, Cook DJ, Wall RJ, Angus DC, Bion J, Kacmarek R, et al. Intensive care unit quality improvement: a "how-to" guide for the interdisciplinary team. Crit Care Med. 2006;34:211-8.

2. Dellinger RP, Carlet JM, Masur H, Gerlach H, Calandra T, Cohen J, et al. Surviving Sepsis Campaign guidelines for management of severe sepsis and septic shock. Crit Care Med. 2004;32:858-73.

3. American Thoracic Society. Guidelines for the management of adults with hospital-acquired, ventilator-associated, and healthcare-associated pneumonia. Am J Respir Crit Care Med. 2005;171:338-416.

4. Brower RG, Rubenfeld G. Lung-protective ventilation strategies in acute lung injury. Crit Care Med. 2003;31:S312-6.

5. Carr JM, Sellke FW, Fey M, Doyle MJ, Krempin JA, de la Torre R, et al. Implementing tight glucose control after coronary artery bypass surgery. Ann Thorac Surg. 2005;80:902-9.

6. Hemmila MR, Rowe SA, Boules TN, Miskulin J, McGillicuddy JW, Schuerer DJ, et al. Extracorporeal life support for severe acute respiratory distress syndrome in adults. Ann Surg. 2004;240:595-605.

7. Ronco C, Bellomo R, Homel P, Brendolan A, Dan M, Piccini P, et al. Effects of different doses in continuous veno-venous haemofiltration on outcomes of acute renal failure: a prospective randomized trial. Lancet. 2000;356:26-30.

8. Fattouch K, Sbraga F, Bianco G, Speziale G, Gucciardo M, Sampognaro R, et al. Inhaled prostacyclin, nitric oxide, and nitroprusside in pulmonary hypertension after mitral valve replacement. J Card Surg. 2005;20:171-6

9. Morales DLS, Gregg D, Helman DN, Williams MR, Naka Y, Landry DW, et al. Arginine vasopressin in the treatment of 50 patients with postcardiotomy vasodilatory shock. Ann Thorac Surg. 2000;69:102-6. 